\title{
Rentabilidad y eficiencia de las entidades financieras de economía social en España
}

\author{
Sanchis Palacio, Joan Ramon* \\ Melián Navarro, Amparo**
}

\section{Resumen}

Las entidades financieras de la Economía Social en España (cajas de ahorros, cooperativas de crédito y secciones de crédito de cooperativas), a la vez que mantienen su carácter social diferenciador, necesitan mejorar sus niveles de eficiencia. El objetivo principal es realizar un análisis comparativo de la rentabilidad y la eficiencia de estas entidades, atendiendo a diversos indicadores, durante el período 2002-2007. La metodología ha consistido en la realización de un estudio empírico basado en el tratamiento estadístico de sus estados contables, a través de las estadísticas oficiales del Banco de España y los resultados obtenidos de un trabajo de campo mediante cuestionario. El análisis demuestra que las cajas de ahorros son las más rentables y eficientes, aunque tanto las cooperativas de crédito como sobre todo las secciones de crédito de cooperativas han mejorado sus niveles, produciéndose un acercamiento entre las tres clases de entidades. En conclusión, estas entidades están realizando un importante esfuerzo de adaptación al entorno bancario, que tendrán que seguir desarrollando durante los próximos años.

Palabras clave: Economía social, cooperativas, eficiencia, rentabilidad, entidades financieras.

\section{Yield and Efficiency of the Financial Organizations of Social Economy in Spain}

\begin{abstract}
The financial organizations of the Social Economy in Spain (savings banks, cooperatives of credit and sections of credit of cooperatives), at the same time as they maintain his character social differentiator, need to improve its levels of efficiency. The primary target is to realise a comparative analysis of the yield and the efficiency of this organizations, taking care of diverse indicators, during

Recibido: 30-01-08・ Aceptado: 02-03-08

* Profesor Titular del Instituto Universitario de Economía Social y Cooperativa de la Universidad de Valencia. E-mail: joan.r.sanchis@uv.es

** Catedrática de Escuela Universitaria del Departamento de Economía Agroambiental de la Universidad Miguel Hernández de Elche. E-mail: amparo.melian@umh.es
\end{abstract}


period 2002-2007. The methodology has consisted of the accomplishment of an empirical study based on the statistical treatment of its countable states, through the official statistics of the Bank of Spain and the obtained results of a work of field by means of questionnaire. The analysis demonstrates that the savings banks are most profitable and efficient, although as much the credit cooperatives as mainly the sections of credit of cooperatives have improved their levels, taking place an approach between the three classes of organizations. In conclusion, this organizations are realising an important effort from adaptation to the banking surroundings, that they will have to continue developing during the next years.

Key words: Social economy, cooperatives, efficiency, yield, financial entities.

\section{Introducción}

La intensa dinámica de cambio que caracteriza el entorno económico actual plantea importantes desafíos a las entidades financieras, y por extensión a las entidades financieras de la Economía Social, al estar sometidas a las tensiones del mercado. El empleo productivo de recursos se asocia con la supervivencia de una empresa, presentando ventajas competitivas con respecto al resto, cuando en el desarrollo de su actividad obtienen mejores resultados.

Las entidades de crédito de la Economía Social (cajas de ahorros, cooperativas de crédito y secciones de crédito de cooperativas) combinan una doble faceta, la financiera y la social (Chaves y Soler, 2005). Por lo que se refiere a la faceta financiera, las cajas de ahorros y las cooperativas de crédito compiten, junto con los bancos, en igualdad de condiciones en un mercado altamente competitivo desde que iniciaron el proceso de homogeneización con la Ley 2/1962, de 14 de abril, de ordenación del crédito y la banca.

Por otra parte, las cajas de ahorros, las cooperativas de crédito y las secciones de crédito de las cooperativas, como entidades de Economía Social (Sanchis y
Palomo, 1997; Sanchis, 2001), presentan peculiaridades propias entre las que destaca como elemento básico su carácter social. Así, por una parte, tratan de evitar y reducir la exclusión financiera de determinados colectivos menos atractivos para el negocio bancario, ratificando su capacidad de inclusión financiera, definida como la aptitud de una entidad para conectar con los circuitos financieros a determinados agentes económicos, que por diversas razones (económicas, distancia, sexo, etc.) se hallan excluidos de éstos. Según Chaves y Soler (2004), este concepto es inherente a las cooperativas de crédito y a las cajas de ahorros, pues su labor ha permitido históricamente conectar la capacidad o la necesidad de financiación de determinados sectores con los circuitos financieros de la economía, lo que ha contribuido a su dinamización y su vinculación con el resto de sectores. Otros estudios como el de Palomo y Valor (2001), constatan una especialización en la financiación de los estratos de renta baja (43\%) y media-baja (37\% del total de sus clientes), mientras que los grupos de renta media-alta y alta suponen un porcentaje de clientes inferior (del $12 \%$ y $5 \%$ respectivamente). Es por ello que estos tres tipos de entidades asumen 
Rentabilidad y eficiencia de las entidades financieras de economía social en España Sanchis Palacio, Joan Ramon y Melián Navarro, Amparo

un compromiso social con parte de los excedentes generados en su actividad financiera, acorde con su personalidad.

Las cajas de ahorros se constituyen como fundaciones, por lo que no tienen títulos de propiedad definidos. Su objeto no consiste per se en la rentabilización de unas inversiones realizadas, sino que el beneficio obtenido se debe destinar a asegurar su supervivencia y a desarrollar la sociedad en la que se encuentran inmersas, es decir, aportar el llamado "dividendo social" (Chaves y Soler, 2005).

Las cooperativas de crédito tienen como objeto básico la prestación de servicios financieros a socios y terceros. Por su personalidad cooperativa, los resultados no pueden distribuirse en función de los desembolsos de capital, por lo que los excedentes se destinan principalmente a la dotación de reservas, al cumplimiento de los principios cooperativos o a la promoción social del entorno.

Las secciones de crédito son departamentos organizados constituidos en el seno de una cooperativa, y con objetivos particulares propios, que desarrollan la actividad financiera de la misma canalizando los flujos financieros socio-sección y sociedad-sección (Melián, Palomo, Sanchis y Soler, 2006). Aunque están excluidas del sistema financiero (Real Decreto 2680/78), han sido unos instrumentos básicos de financiación del medio rural al captar depósitos de los socios y otras secciones de la cooperativa y destinarlos a la inversión crediticia a los propios socios y otras secciones, a la colocación de tesorería en intermediarios financieros o a la adquisición de títulos valores (Campos, Fajardo y Sanchis, 2006). Como secciones de una cooperativa, los resultados de la misma se integran en el global de la cooperativa y de éstos una parte se destinan a reservas, al cumplimiento de los principios cooperativos o a la promoción social.

Pero estas entidades, además de servir a los fines de sus socios y de la sociedad en general, necesitan ser competitivas (rentables y eficientes); si no son competitivas no podrán cumplir con sus fines sociales. Es por ello que es fundamental para su supervivencia la producción a menor coste, es decir, la gestión eficiente de sus recursos (Server y Melián, 2001). El objetivo de este trabajo es analizar la evolución experimentada por las entidades financieras de la Economía Social en España en cuanto a sus niveles de eficiencia y rentabilidad en la actividad de intermediación financiera, basándonos en datos oficiales y en un estudio empírico. El estudio permite realizar un análisis comparativo de los niveles de eficiencia y rentabilidad experimentados por estas entidades durante el periodo 2002-2007.

El trabajo cubre un vacío investigador en el estudio y medición de la eficiencia aplicada a las entidades financieras de Economía Social en España, ya que aunque en el caso de los bancos y las cajas de ahorro se han publicado numerosos trabajos (Pérez y Quesada, 1992; Maroto 1994; Santamaría y Espitia, 1994; Termes, 1994; Pérez y Pastor, 1994; Quesada, 1994; Maroto 1995; Pereira y Cortes, 1998; Pastor 1999; Revell, 2000; Lozano, 2001; Pérez y Maudos, 2001; Tortosa, 2003), los estudios en cooperativas de crédito han sido mucho más escasos (Calvo y González, 1999; Marco y Moya, 2000; Ballestero y Ballbé, 2000; 
Belmonte Ureña 2007) y los aplicados a las secciones de crédito de cooperativas son casi inexistentes (Server y Melián 1999; Server y Melián 2001).

\section{Posición competitiva de las entidades financieras de la Economía Social}

Según el Banco de España, en el año 2007 el sistema bancario español estaba constituido por 140 bancos, 47 cajas de ahorro y 82 cooperativas de crédito ${ }^{1}$. Las más extendidas territorialmente en cuanto a oficinas son las cajas de ahorro con 24.591, seguidos de los bancos con 15.542 y las cooperativas de crédito con 4.953.

Las entidades objeto de nuestra investigación, por tanto, corresponden a 129 entidades (47 cajas de ahorros y 82 cooperativas de crédito). Además, hay que añadir las 320 secciones de crédito de cooperativas agrarias existentes en España ${ }^{2}$.

Las cifras de actividad registradas por cada una de estas entidades durante el periodo estudiado (2002-2007) aparecen recogidas en la Tabla 1.

Las entidades con más peso dentro del conjunto del sistema bancario español a 31 de diciembre de 2007 , son las cajas de ahorros, pues cuentan con el $51 \%$ de los depósitos, el $49 \%$ de los créditos y el $54 \%$ de las oficinas. El peso de las cooperativas de crédito es significativamente inferior en comparación con las cajas de ahorros y los bancos, ya que sus porcentajes alcanzan el $6 \%$ de los depósitos, el $5 \%$ de los créditos y el $11 \%$ de las oficinas. El porcentaje de las secciones de crédito es irrelevante, pues es del $0,2 \%$ de los depósitos, del $0,03 \%$ de los créditos y del $0,7 \%$ de las oficinas. Las entidades financieras de la Economía Social (cajas de ahorros, cooperativas de crédito y secciones de crédito de cooperativas) representan el $57,2 \%$ de los depósitos, el $54 \%$ de los créditos y el $65,7 \%$ de las oficinas del conjunto del sector bancario español.

Las secciones de crédito, aunque realizan una función de intermediario financiero al igual que lo hacen las entidades financieras, no tienen personalidad jurídica propia, si no que son una sección o departamento de una cooperativa, por lo que su actividad financiera se dirige exclusivamente al resto de secciones de la cooperativa y a sus socios. Por lo tanto, la sección de crédito no es un fin en sí mismo sino un instrumento al servicio de la cooperativa y de sus socios, de manera que su objetivo principal ha de ser ofrecer un servicio de calidad, esto es, un servicio hecho a la medida, capaz de atender las necesidades financieras de sus clientes con el mínimo coste posible. Este hecho,

1 Bancos, cajas de ahorros y cooperativas de crédito están reconocidas legalmente como las tres clases de entidades de crédito que pueden operar captando depósitos de terceros.

2 Esta cifra se ha obtenido de un estudio empírico realizado durante el año 2008 por uno de los autores de este trabajo, financiado por la Confederación de Cooperativas Agrarias de España y corresponde a aquellas entidades que están federadas. 
Rentabilidad y eficiencia de las entidades financieras de economía social en España Sanchis Palacio, Joan Ramon y Melián Navarro, Amparo

Tabla 1

Cifras de actividad de las entidades de crédito en España (millones de euros)

\begin{tabular}{|c|c|c|c|c|c|c|c|c|c|c|c|c|}
\hline 00000 & & & & & & & & & & & & \\
\hline 800000 & & & & & 周 & 凨 & & & & & & \\
\hline 700000 & & & & & 国 & 閆 & & & & & & \\
\hline 600000 & & & & & & & & & & & & \\
\hline 500000 & & 党 & & & & & & & & & & \\
\hline 400000 & & 葫 & & & 角 & & & & & & & \\
\hline 300000 & & 䦥 & & & 闕 & . & & & & & & \\
\hline 200000 & $\omega_{1}$ & 閸 & & & 閶 & & & & & & & \\
\hline 100000 & 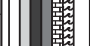 & 監 & - & & 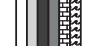 & & 国 & & & & & \\
\hline 0 & $\begin{array}{l}\text { Dep } \\
\text { Bcos }\end{array}$ & $\begin{array}{l}\text { Dep } \\
\text { CA }\end{array}$ & $\begin{array}{l}\text { Dep. } \\
\text { CC }\end{array}$ & $\begin{array}{l}\text { Dep } \\
\text { SC }\end{array}$ & $\begin{array}{l}\text { Ctos } \\
\text { Bcos }\end{array}$ & $\begin{array}{c}\text { Ctos } \\
\text { CA }\end{array}$ & $\begin{array}{c}\text { Ctos } \\
\text { CC }\end{array}$ & $\begin{array}{c}\text { Ctos } \\
\text { SC }\end{array}$ & $\begin{array}{c}\text { Of } \\
\text { Bcos }\end{array}$ & Of $\mathrm{CA}$ & Of CC & Of SC \\
\hline$\square 2002$ & 267604 & 323977 & 40525 & 760 & 341250 & 0315846 & 36084 & 236 & 14072 & 20326 & 4275 & 360 \\
\hline$\square 2003$ & 270414 & 359531 & 44452 & 767 & 383623 & 3368457 & 42319 & 243 & 14074 & 20871 & 4460 & 350 \\
\hline$\square 2004$ & 300180 & 413872 & 51328 & 946 & 439851 & 1444478 & 49911 & 273 & 14168 & 21503 & 4559 & 343 \\
\hline$\square 2005$ & 352007 & 429607 & 56404 & 1027 & 551934 & 4567742 & 64968 & 276 & 14533 & 22410 & 4656 & 337 \\
\hline 圄2006 & 424583 & 502688 & 65535 & 2108 & 681386 & 6723143 & 78449 & 554 & 15096 & 23418 & 4772 & 324 \\
\hline 2007 & 468514 & 565500 & 71960 & 2000 & 792059 & 9847262 & 91366 & 500 & 15542 & 24591 & 4953 & 320 \\
\hline
\end{tabular}

Fuente: elaboración propia a partir de datos del Banco de España (2007).

unido a las importantes limitaciones que presentan en cuanto a su operatoria, condiciona sus niveles de rentabilidad y de eficiencia.

Por lo tanto, para proceder a medir los niveles de rentabilidad y eficiencia de las secciones de crédito, habrá que tener en cuenta estos aspectos. Los criterios tradicionales más utilizados como ratios de eficiencia bancaria son los Gastos de Explotación por Activos Totales (GE/ AT) y los Gastos de Explotación por el Margen Ordinario (GE/ MO) y como ratios de rentabilidad, el ROA o rentabilidad del activo (Beneficio antes de impuestos/ Activos Totales Medios) y el ROE o rentabilidad de los recursos propios (Beneficio antes de impuestos/ Recursos Propios) (Pérez y Doménech, 1990; Termes 1994; De la Cuesta, 1996; Pereira y Cortés,
1998; Pérez, Maudos y Pastor, 1999; Fuentes Egusquiza, 2003). No se incluyen las variables depósitos por oficina y créditos por oficina (que también son utilizadas en los estudios sobre rentabilidad de las entidades de crédito), porque se está realizando un estudio sobre intermediarios financieros con una única oficina.

También ha sido habitual medir la rentabilidad y la eficiencia de las entidades de crédito "cotejando los tipos de interés que las entidades fijan en sus operaciones con sus clientes a los créditos concedidos y los depósitos recibidos" (Server y Melián, 1999: 63). En el trabajo de Server y Melián (1999) se analiza para las secciones de crédito valencianas la productividad que la gestión de sus activos rentables les ocasiona y la remuneración que realizan de sus depósitos. Los 
autores consideran como activos rentables las partidas del activo del balance (tesorería y otros productos financieros en entidades de crédito, inversión crediticia y cartera de títulos), de manera que para determinar la rentabilidad de dichos activos, éstos se relacionan con las partidas de la cuenta de pérdidas y ganancias (productos de inversión crediticia, productos de tesorería e intermediarios financieros y productos de la cartera de títulos). Por otra parte, el coste del pasivo está compuesto por los depósitos en cuentas corrientes, cuentas de ahorro e imposiciones a plazo; y su determinación se realiza comparando la partida Intereses de acreedores del debe de la cuenta de pérdidas y ganancias con la partida acreedores del pasivo del balance de situación. Finalmente, para contrastar ambas medidas (rentabilidad de los activos y coste del pasivo) se obtiene un estimador o índice global como es el diferencial financiero, el cual mide la diferencia entre la rentabilidad del producto del activo financiero y el coste del pasivo.

Frente a los estudios sobre eficiencia que trabajan sobre ratios, existen otros criterios de medida de la rentabilidad y la eficiencia más sofisticados que son los modelos multicriterio, entre los que se incluye la estimación de fronteras eficientes y la evaluación de las ineficiencias tipo X (Belmonte Ureña, 2007). Los modelos multicriterio, como señala $\mathrm{Ba}$ llestero (2000: 25), "se caracterizan por su versatilidad para reflejar todo un conjunto de detalles que la empresa debe tener en mente a la hora de decidir sobre un proyecto"; y entre ellos se incluye la estimación de fronteras eficientes como un modelo multiobjetivo útil para estimar va- riables tales como la rentabilidad y el riesgo. Ballestero y Ballbé (2000) utilizan una variante de la estimación de fronteras eficientes, el Modelo Data Envelopement Análisis (DEA), que clasifica las actividades empresariales según su eficiencia global y según su eficiencia técnica, aplicado a los índices de eficiencia de las cooperativas de crédito y cajas rurales. Las ineficiencias tipo $X$ hacen referencia a las deficiencias en la gestión y organización de los recursos y capacidades de la organización y, por tanto, son producto de una incorrecta formulación y/o implementación de la estrategia; el problema básico consiste en establecer un buen ajuste entre la estrategia a seguir por la empresa y la estructura organizativa que ésta utiliza para ejecutar la estrategia, pero puesto que diferentes estrategias requieren diferentes formas de organizar la tecnología, diferentes estructuras, procesos, diseño de puestos, ordenación de incentivos y políticas de personal, no es fácil conseguir el ajuste necesario. En el sector bancario, diferentes estudios realizados han demostrado que los niveles de eficiencia tipo $X$ son mucho más rentables y efectivos para las entidades de crédito que las eficiencias basadas en las economías de escala y aumento de tamaño (Altumbas et al., 1998; Pérez, Maudos y Pastor, 1999). La necesidad de obtener información detallada y homogénea para la aplicación de estos modelos que no ha sido facilitada por las secciones de crédito, nos ha hecho descartar su utilización en el presente estudio, si bien se podría plantear la realización de un nuevo estudio más detallado en el sentido apuntado por dichos criterios. 
Rentabilidad y eficiencia de las entidades financieras de economía social en España Sanchis Palacio, Joan Ramon y Melián Navarro, Amparo

En definitiva, y considerando la información disponible, se ha decidido medir los niveles de rentabilidad y eficiencia, atendiendo a los criterios clásicos utilizados por numerosos autores en el ámbito del sector financiero. En este sentido, el análisis se ha estructurado de la siguiente manera: Análisis de las ratios de rentabilidad (ROA y ROE) y de eficiencia (GE/AT y GE/MO), más utilizados en el sector bancario en España; y Análisis de los ratios de rentabilidad y eficiencia siguiendo el modelo planteado por Server y Melián (1999), diferencial financiero entre la rentabilidad de los activos y los costes del pasivo.

En ambos casos se procederá a realizar un análisis comparativo con las cajas rurales y las cajas de ahorro, por ser éstas las entidades de crédito que compiten directamente en el segmento de mercado en el cual desarrollan su actividad financiera las secciones de crédito.

Las fuentes de información empleadas son por una parte, los datos estadísticos proporcionados por las instituciones correspondientes, el Banco de España, la Confederación Española de Cajas de Ahorro, la Unión Nacional de Cooperativas de Crédito, el Instituto Valenciano de Finanzas, Instituto Catalán de Finanzas, en sus informes anuales; y, por otra, los documentos contables anuales (balance y cuenta de resultados) de cada una de las secciones de crédito objeto de estudio. En este último caso es importante señalar que el estudio se refiere a 178 secciones de crédito de las cuales se ha podido obtener la información contable que ellas mismas han proporcionado, esto es, casi el $70 \%$ de la muestra utilizada en el estudio (258 entidades) y alrededor del $50 \%$ de la población total (320 entidades).

\section{Análisis de la rentabilidad y la eficiencia de las entidades financieras de la Economía Social}

A continuación se analizan los resultados obtenidos de la investigación realizada diferenciando entre el análisis de la rentabilidad, el análisis de la eficiencia y el análisis del diferencial financiero, que son los tres indicadores que se han utilizado en el estudio para describir las principales diferencias entre los tres tipos de entidades que constituyen las entidades financieras de la Economía Social en España.

En primer lugar, se han calculado las ratios de rentabilidad de los activos (ROA) y de los recursos propios (ROE) de las entidades financieras de la Economía Social en España, tal como muestra la Tabla 2.

En general, se observa que las entidades más rentables son las cajas de ahorros, tanto en lo que se refiere a la rentabilidad de los activos $(0,95 \%)$ como a la rentabilidad de los recursos propios $(22,83 \%)$. Si se comparan las cooperativas de crédito con las secciones de crédito de las cooperativas, se puede ver que las primeras son más rentables según $\mathrm{ROA}$ pero menos rentables según ROE.

La comparativa referida a la ROA se observa en el Gráfico 1, donde se aprecia que si bien los niveles de rentabilidad de las secciones de crédito de las cooperativas agrarias de España son inferiores a los de las cooperativas de crédito y cajas de ahorros, las diferencias han ido reduciéndose con el paso del tiempo, dado que la tasa de crecimiento de la rentabilidad de las secciones es su- 
Tabla 2

Ratios de rentabilidad de las entidades financieras de la Economía Social en España durante el período 2002-2007

\begin{tabular}{|c|c|c|c|c|c|c|c|c|}
\hline \multirow[t]{2}{*}{ Años } & \multicolumn{2}{|c|}{$\begin{array}{c}\text { Cajas } \\
\text { de Ahorros }\end{array}$} & \multicolumn{2}{|c|}{$\begin{array}{c}\text { Cooperativas } \\
\text { de Crédito }\end{array}$} & \multicolumn{2}{|c|}{$\begin{array}{l}\text { Secciones } \\
\text { de Crédito }\end{array}$} & \multicolumn{2}{|c|}{$\begin{array}{c}\text { Valor } \\
\text { medio total }\end{array}$} \\
\hline & $\mathrm{ROA}$ & ROE & ROA & ROE & ROA & ROE & ROA & ROE \\
\hline 2002 & 0.84 & 14.78 & 0.82 & 9.77 & 0.23 & 2.68 & 0.63 & 9.08 \\
\hline 2003 & 0.84 & 15.09 & 0.79 & 9.65 & 0.34 & 4.04 & 0.66 & 9.61 \\
\hline 2004 & 0.84 & 15.57 & 0.80 & 9.75 & 0.53 & 6.71 & 0.72 & 10.68 \\
\hline 2005 & 0.77 & 14.00 & 0.84 & 9.89 & 0.54 & 6.74 & 0.72 & 10.21 \\
\hline 2006 & 0.91 & 17.22 & 0.73 & 9.11 & 0.56 & 6.81 & 0.73 & 11.05 \\
\hline 2007 & 0.95 & 22.83 & 0.74 & 9.73 & 0.58 & 7.03 & 0.76 & 13.20 \\
\hline
\end{tabular}

Fuente: Elaboración propia a partir de datos del Banco de España (2007).

\section{Gráfico 1}

Evolución comparativa de la ROA de las entidades financieras de la Economía Social en España durante el periodo 2002-2007

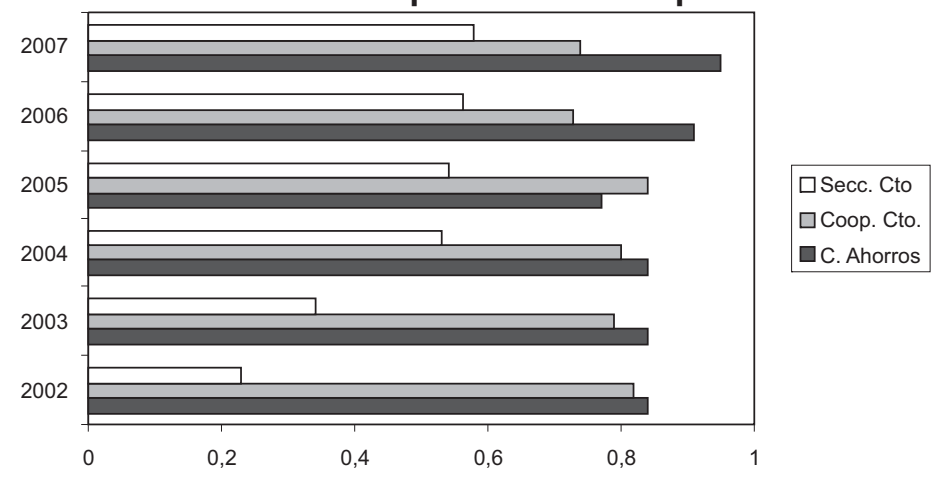

Fuente: Elaboración propia (2007).

perior a la de las cajas de ahorros y cajas rurales en este periodo. Así, mientras la rentabilidad de las secciones de crédito se ha incrementado desde el $0,23 \%$ hasta un nivel próximo al $0,6 \%$, las rentabilidades de las cooperativas de crédito y las cajas de ahorros se han mantenido en un nivel cercano al 0,8-0,9\%. Aunque las cajas de ahorros han mejorado su rentabilidad durante el período estudiado (han se- guido una tendencia descendente hasta 2005, año a partir del cual comienzan a recuperarse), las cooperativas de crédito han seguido una tendencia descendente a lo largo de todo el período.

En términos comparativos, según muestra el Gráfico 2, la rentabilidad media de los recursos propios de las secciones de crédito está por debajo de la media de las cooperativas de crédito y las 
Rentabilidad y eficiencia de las entidades financieras de economía social en España Sanchis Palacio, Joan Ramon y Melián Navarro, Amparo

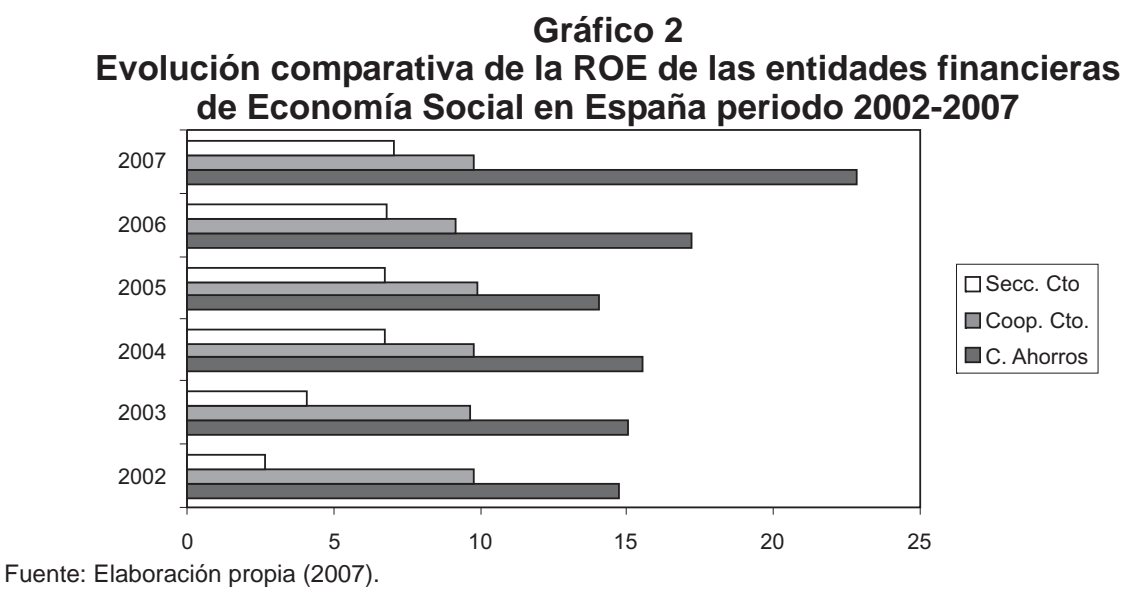

cajas de ahorros; aunque sus elevadas tasas de crecimiento experimentadas durante el período considerado, han permitido reducir de manera significativa el diferencial con respecto a éstas. La tendencia es muy similar a lo observado en el gráfico anterior referido a las rentabilidades del activo.

En segundo lugar, se han calculado las ratios de eficiencia de los activos (GE/AT) y del margen ordinario (GE/MO) de las entidades financieras de la Economía Social en España, tal como muestra la Tabla 3.

Las ratios indicativos de la eficiencia muestran por una parte una evolución positiva en todas las entidades, dado que la disminución de la ratio indica una mejora de la misma, y por otra que las secciones de crédito son casi tan eficientes como las cajas de ahorros y más eficientes que las cooperativas de crédito si se evalúa según el cociente gastos de explotación respecto al activo total. No se obtiene el mismo resultado cuando se mide con respecto al margen ordinario, siendo las cajas de ahorros las entidades más eficientes, lo que también puede deberse a que en las entidades de crédito cooperativo no es frecuente el cobro de comisiones y por lo tanto la partida de otros productos ordinarios que integra los ingresos derivados de comisiones y otros servicios es muy baja. Otras entidades como éstas sí pueden recuperar parte de la disminución del margen de intermediación como consecuencia del mayor ajuste entre los tipos de interés de las partidas de activo y de pasivo.

El Gráfico 3 muestra el comparativo entre secciones de crédito de cooperativas, cooperativas de crédito y cajas de ahorros para la eficiencia referida a los activos totales. Los niveles de ineficiencia de las cajas rurales se sitúan en unos porcentajes próximos al $2 \%$ mientras que en las secciones de crédito y cajas de ahorros se sitúan por debajo del 1,5\%. Se deduce por ello, que las secciones de crédito son más eficientes que las cooperativas de crédito y ligeramente menos eficientes que las cajas de ahorros. 
Tabla 3

Ratios de eficiencia de las entidades financieras de la Economía Social en España durante el período 2002-2007

\begin{tabular}{ccccccccc}
\hline \multirow{2}{*}{ Años } & \multicolumn{2}{c}{$\begin{array}{c}\text { Cajas } \\
\text { de Ahorros }\end{array}$} & \multicolumn{2}{c}{$\begin{array}{c}\text { Cooperativas } \\
\text { de Crédito }\end{array}$} & \multicolumn{2}{c}{$\begin{array}{c}\text { Secciones } \\
\text { de Crédito }\end{array}$} & \multicolumn{2}{c}{$\begin{array}{c}\text { Valor } \\
\text { medio total }\end{array}$} \\
\cline { 2 - 9 } & GE/AT & GE/MO & GE/AT & GE/MO & GE/AT & GE/MO & GE/AT & GE/MO \\
\hline 2002 & 1.74 & 56.40 & 2.54 & 63.55 & 1.37 & 90.09 & 1.88 & 70.01 \\
2003 & 1.66 & 54.56 & 2.43 & 63.30 & 1.40 & 86.75 & 1.83 & 68.20 \\
2004 & 1.54 & 53.98 & 2.28 & 63.95 & 1.27 & 84.92 & 1.70 & 67.62 \\
2005 & 1.32 & 54.01 & 2.04 & 66.05 & 1.22 & 82.71 & 1.53 & 67.59 \\
2006 & 1.16 & 46.95 & 1.81 & 61.81 & 1.21 & 81.67 & 1.39 & 63.48 \\
2007 & 1.12 & 43.41 & 1.71 & 56.97 & 1.20 & 80.89 & 1.34 & 60.42 \\
\hline
\end{tabular}

Fuente: Elaboración propia a partir de datos del Banco de España (2007).

\section{Gráfico 3}

Evolución comparativa de la eficiencia (GE/AT) de las entidades financieras de la Economía Social en España durante periodo 2002-2007

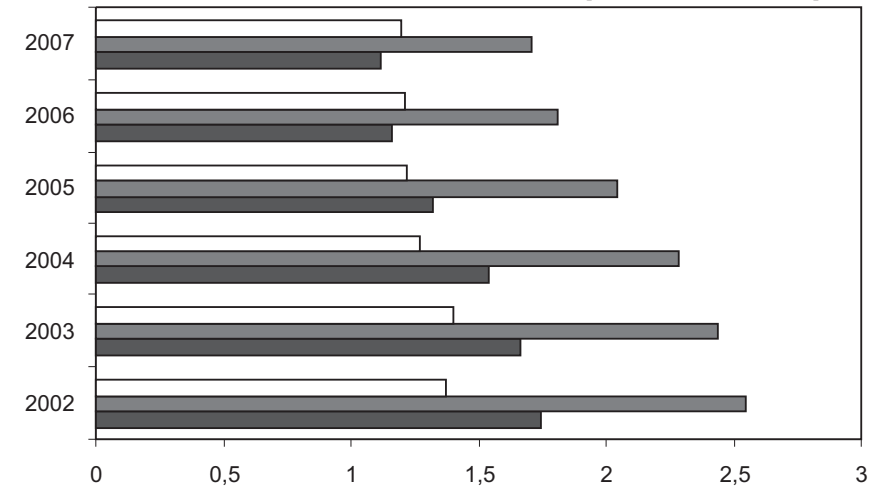

En comparación con las cooperativas de crédito y cajas de ahorro con respecto a la eficiencia según margen ordinario, según se refleja en el Gráfico 4, se observa una mayor eficiencia por parte de las entidades de crédito (cajas de ahorros y cooperativas de crédito) sobre las secciones de crédito, si bien también se observa un ligero aumento en las tasas de eficiencia de és- tas últimas, que les ha permitido reducir la diferencia, sobre todo con respecto a las cooperativas de crédito.

En tercer y último lugar, se ha calculado el diferencial financiero (la diferencia entre el producto total del activo productivo y el coste del pasivo) de las entidades financieras de la Economía Social en España, tal como muestra la Tabla 4. 
Rentabilidad y eficiencia de las entidades financieras de economía social en España Sanchis Palacio, Joan Ramon y Melián Navarro, Amparo

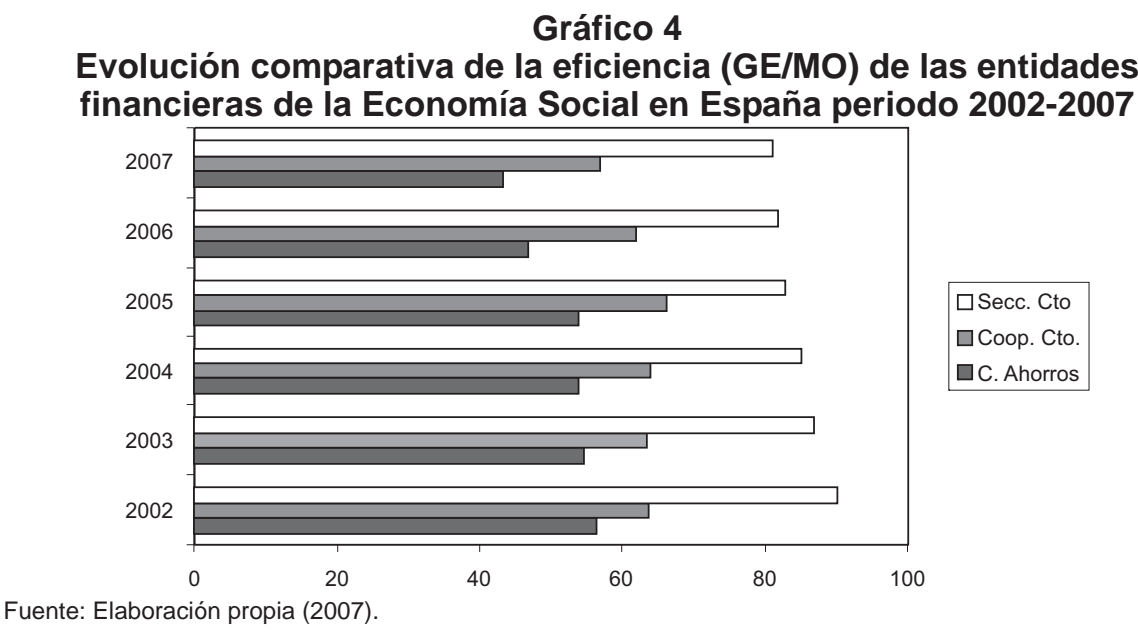

Tabla 4

Diferencial financiero de las entidades financieras de la Economía Social en España durante el período 2002-2007

\begin{tabular}{ccccc}
\hline Años & Cajas Ahorros & Coop. Crédito & Secc. Crédito & Valor medio \\
\hline 2002 & 3.05 & 4.40 & 1.12 & 2.86 \\
2003 & 2.70 & 3.83 & 1.13 & 2.55 \\
2004 & 2.26 & 3.22 & 1.36 & 1.28 \\
2005 & 1.29 & 2.45 & 1.19 & 1.64 \\
2006 & 0.73 & 2.35 & 0.47 & 1.18 \\
2007 & 1.93 & 1.72 & 0.47 & 1.37 \\
\hline
\end{tabular}

Fuente: Elaboración propia a partir de datos del Banco de España (2007).

Las entidades que presentan un mayor diferencial son las cajas de ahorros, con un valor próximo al $2 \%$, seguidas de las cooperativas de crédito, las cuales alcanzan un valor superior al $1,5 \%$. Según esta medida, las secciones de crédito se sitúan en una posición de mayor debilidad, ya que su diferencial apenas llega al 0,5\%.

Sin embargo, si se analiza la evaluación seguida por dichos diferenciales durante el período estudiado (según muestra el Gráfico 5), se observa que tanto las cajas de ahorros como las cooperativas de crédito han sufrido un descenso significativo (de un punto en el caso de las cajas de ahorros y de más de dos puntos en el caso de las cooperativas de crédito); mientras que las secciones de crédito han aguantado mejor el descenso al perder poco más de medio punto.

La disminución producida a lo largo del período considerado del diferencial financiero en las tres entidades analizadas 


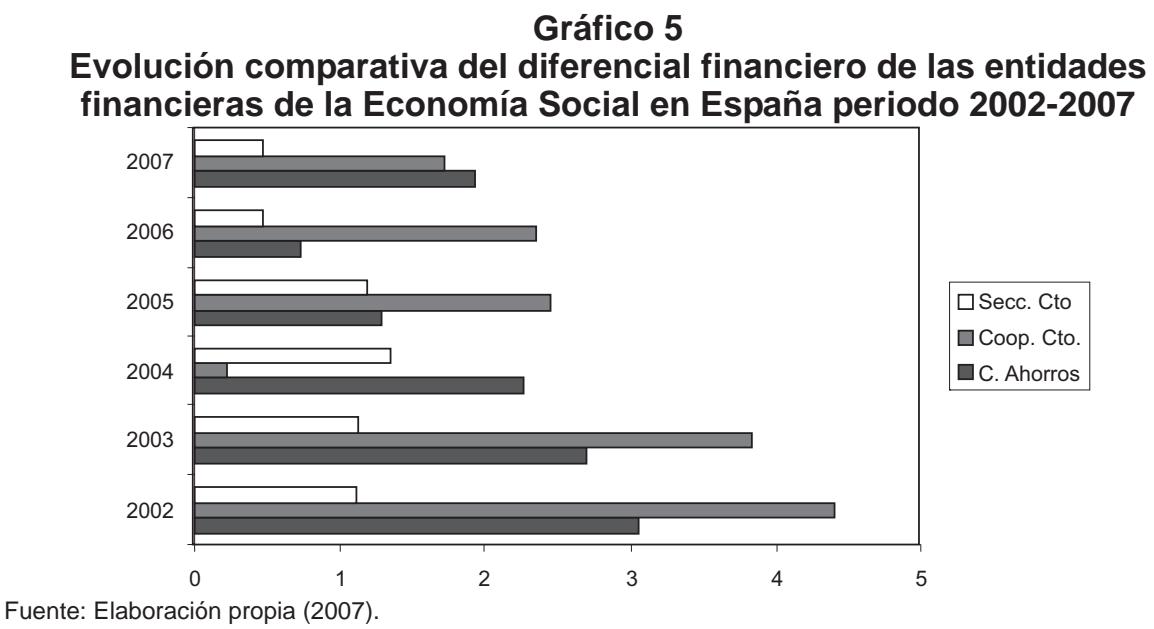

se explica por el estrechamiento de los márgenes que se ha producido en el sector bancario en España: por una parte, por la disminución de los tipos de interés de la operaciones de activo (reducción del volumen de productos del activo); y por otra, por el aumento en los tipos de interés de las operaciones de pasivo (incremento del volumen de costes del pasivo). Además, el menor diferencial financiero que muestran las secciones de crédito en comparación con las cooperativas de crédito es debido a la función social que desempeñan estas entidades al ofrecer operaciones de activo a unos tipos de interés inferiores a los del mercado para así satisfacer las necesidades de los socios de las cooperativas. Téngase en cuenta que el objetivo de la sección de crédito es un objetivo más social que empresarial, ya que son creadas por las cooperativas agrarias para cubrir las necesidades financieras de sus otras secciones y de sus socios.
Por todo ello es importante reconocer que debemos ser cautos al estudiar la eficiencia de unas entidades que pertenecen al amplio campo de la Economía Social, con criterios de estricta rentabilidad como si se tratara de sociedades puramente bancarias. El concepto de eficiencia es muy complejo, y en él hay que englobar su carácter multiatributo. De hecho, Azofra (1994) afirma que el éxito o fracaso de una organización depende, en última instancia, de su eficiencia, entendida ésta, si no en un sentido estrictamente económico, sí desde una triple perspectiva: económico-financiera, organizativa y social.

La evolución seguida por el diferencial financiero se puede entender mejor si se analizan las cifras que se recogen en la Tabla 5, referidas tanto al producto de los activos como al coste del pasivo de las tres clases de entidades estudiadas. Los mayores productos del activo corresponden a las cajas de ahorros $(5,37 \%)$ seguidos de las cooperativas de crédito $(3,76 \%)$ y a bastante distancia de las sec- 
Rentabilidad y eficiencia de las entidades financieras de economía social en España Sanchis Palacio, Joan Ramon y Melián Navarro, Amparo

Tabla 5

Producto del activo y Coste del pasivo de las entidades financieras de la Economía Social en España durante período 2002-2007

\begin{tabular}{cccccccccc}
\hline \multirow{2}{*}{ Años } & \multicolumn{2}{c}{$\begin{array}{c}\text { Cajas } \\
\text { de Ahorros }\end{array}$} & \multicolumn{2}{c}{ Cooperativas de Crédito } & \multicolumn{2}{c}{$\begin{array}{c}\text { Secciones } \\
\text { de Crédito }\end{array}$} & \multicolumn{2}{c}{$\begin{array}{c}\text { Valor } \\
\text { medio total }\end{array}$} \\
\cline { 2 - 10 } & Producto & Coste & Producto & Coste & Producto & Coste & Producto & Coste \\
\hline 2002 & 6.08 & 3.03 & 6.38 & 1.99 & 4.22 & 3.09 & 5.56 & 2.70 \\
2003 & 5.06 & 2.36 & 5.37 & 1.54 & 3.91 & 2.78 & 4.78 & 2.23 \\
2004 & 4.29 & 2.04 & 4.49 & 1.27 & 3.16 & 1.79 & 3.98 & 1.70 \\
2005 & 3.51 & 2.22 & 3.83 & 1.39 & 2.68 & 1.48 & 3.34 & 1.70 \\
2006 & 3.80 & 3.07 & 4.17 & 1.82 & 2.02 & 1.56 & 3.33 & 2.15 \\
2007 & 5.37 & 3.45 & 3.76 & 2.04 & 2.01 & 1.54 & 3.71 & 2.34 \\
\hline
\end{tabular}

Fuente: Elaboración propia a partir de datos del Banco de España (2007).

ciones de crédito de las cooperativas $2,01 \%$ ). En lo que se refiere al coste del pasivo (depósitos o cuentas acreedoras), la situación es la misma (mayor en las cajas de ahorros y menor en las secciones de crédito), aunque las diferencias son algo menores.

La mayor labor social realizada por las secciones de crédito al financiar las actividades crediticias de sus socios a unos tipos de interés más bajos se traduce, además de en una rentabilidad del activo baja, en unos menores niveles de rentabilidad en comparación con las entidades de crédito (cooperativas de crédito y cajas de ahorros), tal como muestra el Gráfico 6. En él se aprecia cómo no sólo las rentabilidades de los productos financieros de las secciones de crédito de las cooperativas agrarias de España son menores a las de las entidades de crédito, sino que además dicha diferencia va aumentando con el paso del tiempo, si bien el descenso en dichos niveles de rentabilidad también se da en cooperativas de crédito y cajas de ahorros como conse- cuencia del estrechamiento de los márgenes financieros.

Por último, según muestra el Gráfico 7, las secciones de crédito tienen unos costes de pasivo ligeramente superiores a los de las cooperativas de crédito pero inferiores a los de las cajas de ahorros en la última fase del período analizado. Además, el fuerte descenso experimentado en los costes financieros de las secciones durante el período ha disminuido sensiblemente las diferencias con respecto a las cajas rurales de modo que en el último año prácticamente se igualan.

Así pues, para medir la eficiencia económica existe la serie de variables que se han expuesto que pueden determinar la consecución de los objetivos económico-financieros. La eficiencia organizativa tiene un carácter interno directamente relacionado con el funcionamiento de su estructura, y la eficiencia social intenta expresar el grado de satisfacción de los participantes directos, los socios, de ahí la importancia de la obra social en el ámbito externo de la organización. 


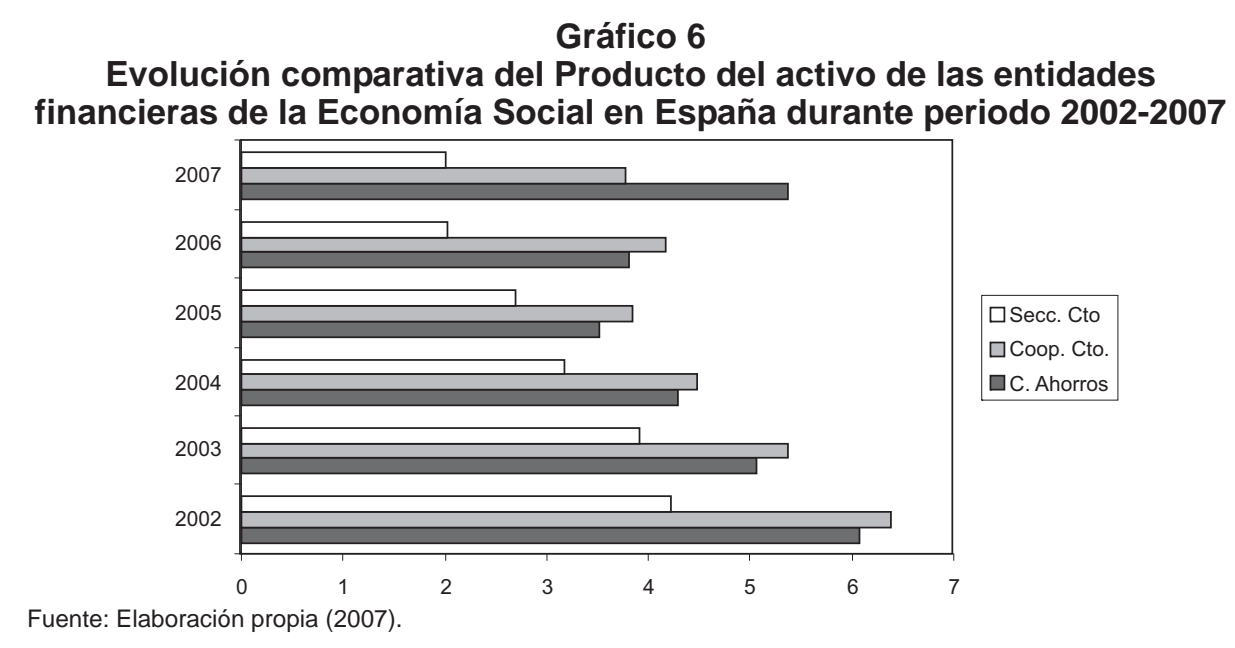

Gráfico 7

Evolución comparativa del Coste del pasivo de las entidades financieras de la Economía Social en España durante periodo 2002-2007

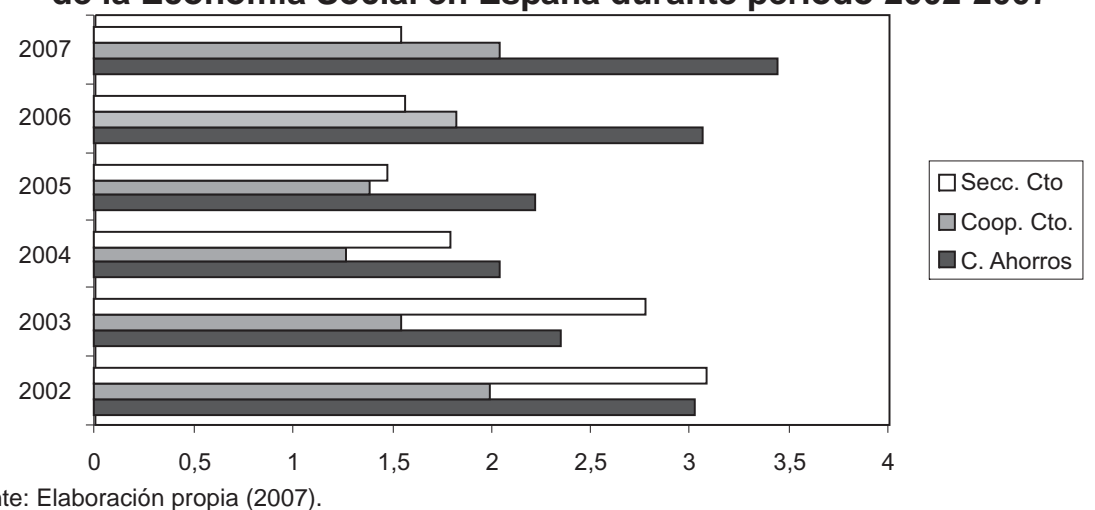

\section{Conclusiones}

Las entidades financieras de la Economía Social desempeñan una labor destacada en la consolidación y el desarrollo de las diferentes iniciativas que surgen dentro de este campo, de manera que ofrecen financiación en unas condiciones más favorables a las del mercado.
En este sentido, cajas de ahorro, cooperativas de crédito y secciones de crédito de cooperativas han contribuido de manera sustancial a la implantación de la Economía Social en España.

Sin embargo, existe una opinión generalizada sobre si estas entidades pueden tener unos niveles de rentabilidad y de eficiencia bajos, como conse- 
Rentabilidad y eficiencia de las entidades financieras de economía social en España Sanchis Palacio, Joan Ramon y Melián Navarro, Amparo

cuencia de la labor social que desempeñan. En este sentido, el trabajo demuestra que a pesar de realizar una función social destacada dentro del ámbito de la Economía Social, son capaces de alcanzar y mantener unos niveles de rentabilidad y de eficiencia altos, similares a los de otras entidades financieras que no pertenecen a la Economía Social, lo que las hace viables y sólidas.

Con el fin de medir los niveles de rentabilidad y de eficiencia económica de las entidades financieras de la Economía Social, en este trabajo se han empleado los ratios de rentabilidad, ROA y ROE, y de eficiencia GE/MO y GE/AT, así como el estudio del diferencial financiero. Los citados indicadores revelan una menor rentabilidad de las secciones de crédito de las cooperativas en España si se comparan con las cifras de cajas de ahorro y cooperativas de crédito (cajas rurales), pero es importante destacar que se aprecia una evolución positiva en los niveles de rentabilidad de las secciones de crédito, pues las diferencias han ido reduciéndose con los años. Lo mismo ocurre cuando se analiza la eficiencia según el ratio GE/MO.

Las secciones de crédito presentan los mejores niveles de eficiencia frente a las otras entidades financieras estudiadas, cajas de ahorros y cooperativas de crédito, cuando se utiliza como indicador el ratio GE/AT. Además y siguiendo en la línea evolutiva de los últimos años han experimentado un ligero aumento durante el período contemplado.

En cuanto al empleo como indicador de la productividad del activo, de nuevo las diferencias de rentabilidad son favorables a las entidades de crédito, es decir, que las cajas de ahorro y las cooperativas de crédito presentan niveles de rentabilidad de los activos superiores a los de las secciones de crédito. Sin embargo, los niveles de rentabilidad de las secciones de crédito se han incrementado sensiblemente durante el período contemplado, con unas tasas de crecimiento superiores a las de cajas de ahorro y cajas rurales; por lo que las diferencias en la rentabilidad de los activos se han reducido de manera significativa. En cuanto al pasivo, las secciones muestran una mayor remuneración por sus depósitos que las cajas rurales y por debajo de las cajas de ahorro. Si ambos indicadores se aglutinan para el cálculo del diferencial financiero, se observa el importante esfuerzo de contención del diferencial en las secciones de crédito que prácticamente se ha mantenido constante en el periodo analizado, frente a la significativa caída de cajas de ahorro y cooperativas de crédito que se ha reducido a la mitad.

Lo anterior son herramientas para cuantificar la eficiencia económica de las secciones, toda vez que su eficiencia social está fuera de toda duda. Además y dado que la sección de crédito es un medio, un instrumento al servicio de la cooperativa, y no un fin en sí misma, el fin es la cooperativa, en ocasiones se supeditan los resultados de la propia sección a los de la cooperativa, enmascarando una mayor eficiencia económica, por ejemplo cuando la sección soporta como propios algunos costes de la cooperativa.

De dicho análisis se puede deducir también que el compromiso social adquirido por este tipo de entidades financieras no compromete su viabilidad, si no que incluso la puede garantizar, ya que su acti- 
vidad se lleva a cabo desde una perspectiva estratégica basada en la diferenciación y el trato personalizado al cliente, lo que les permite ofrecer un servicio de calidad.

Por lo tanto, el análisis demuestra que las cajas de ahorros son las más rentables y eficientes, aunque tanto las cooperativas de crédito como sobre todo las secciones de crédito de cooperativas han mejorado sus niveles, produciéndose un acercamiento entre las tres clases de entidades. En conclusión, estas entidades están realizando un importante esfuerzo de adaptación al entorno bancario, que tendrán que seguir desarrollando durante los próximos años.

\section{Referencias Bibliográficas}

Altumbas, Yolanda; Gardener, Edward; Molyneux, Philip (1998). Efficiency in European Banking. Research $\mathrm{Pa}$ pers in Banking and Finance RP98/2, Institute of European Finance.

Azofra, Valentín (1994). Estructura organizativa y resultados de las cajas de ahorros españolas. Papeles de Economía Española, No 58, pp. 284-296.

Ballestero, Enrique (2000). Modelos multicriterio en la programación de cooperativas. Actas del Primer Congreso sobre Cooperativismo Español, SeviIla, Fundación Fernando Garrido Tortosa, pp. 15-36.

Ballestero, Enrique; Ballbé, Pilar (2000). Índices de eficiencia en cooperativas de crédito y cajas rurales. Actas del Primer Congreso sobre Cooperativismo Español, Sevilla, Fundación Fernando Garrido Tortosa, pp. 373-384.

Banco de España BDE (2008). Boletín Estadístico del Banco de España, Madrid.
Belmonte Ureña; Luis Jesús (2007). El sector de cooperativas de crédito en España. Sevilla, Consejo Económico y Social de Andalucía.

Calvo, Antonio; González, José Ignacio (1999). Eficiencia económica y social de las cooperativas de crédito españolas. Revista de Estudios Cooperativos, Revesco, No 67, pp. 51-70.

Campos, Vanessa; Fajardo, Gemma; Sanchis, Joan Ramon (2006). Triple justificación de las secciones de crédito de las cooperativas agrarias de la Comunidad Valenciana: jurídica, económica y social. Revista CIRIEC-España, No. 54, pp. 126-154.

Chaves, Rafael; Soler, Francisco (2004), EI gobierno de las cooperativas de crédito en España. Valencia, Ed. Ciriec-España.

Chaves, Rafael; Soler, Francisco (2005). EI comportamiento de cajas de ahorros y cooperativas de crédito españolas frente a los fines sociales: la obra benéfico-social y el Fondo de educación y promoción cooperativa. Baeza, $X$ Jornadas de investigadores en Economía Social.

De La Cuesta, María (1996). El control de la gestión en las entidades bancarias. Los modelos tradicionales y el ABM. Madrid, Universidad Nacional de Educación a Distancia.

Fuentes Egusquiza, Ignacio (2003). Un análisis de las fusiones bancarias recientes (1997-2000) en España. Boletín Económico del Banco España, julio-agosto, pp. 71-78.

Instituto Valenciano de Finanzas IVF (20022007). Informe Anual del Instituto Valenciano de Finanzas, Valencia.

Lozano, Ana (2001). La eficiencia del sistema bancario español en el marco de la Unión Europea. Ekonomiaz. Revista Vasca de economía, No. 48, pp. 314-343. 
Rentabilidad y eficiencia de las entidades financieras de economía social en España Sanchis Palacio, Joan Ramon y Melián Navarro, Amparo

Marco, Amparo; Moya, Ismael (2000). El efecto del tamaño en la eficiencia de las cajas rurales españolas. Revista Española de Estudios Agrosociales y Pesqueros, No. 187, pp. 87-108.

Maroto, Juan Antonio (1994). El proceso de concentración de bancos y cajas de ahorro en España. Papeles de Economía Española, No. 58, pp. 88104

Maroto, Juan Antonio (1995). Las cajas de ahorro españolas: competitivas y rentables. Cuadernos de Información Económica, No. 103, pp. 39-49.

Melián, Amparo; Palomo, Ricardo; Sanchis, Joan Ramon, Soler, Francisco (2006), La investigación en materia de cooperativas de crédito y grupos cooperativos. Revista CIRIEC-España. No. 56 , pp. 37-58.

Palomo, Ricardo; Valor; Carmen (2001). Banca cooperativa. Entorno financiero y proyección social. Madrid, Unión Nacional de Cooperativas de Crédito.

Pastor, José Manuel (1999). Credit risk and efficiency in the European banking systems: a three-stage analysis. Documentos de trabajo serie EC, No. 18. Valencia, Instituto Valenciano de Investigaciones Económicas.

Pereira, Jorge Juan; Cortés, Juan (1998). Los resultados de las cajas de ahorro en la década de los noventa. Papeles de economía española, No. 74-75, pp. 36-54.

Pérez, Francisco; Domenech, Rafael (1990). La productividad de los bancos y cajas de ahorro españoles. Madrid, Fundación FIES, Documento de trabajo No. 66 .

Pérez, Francisco; Quesada, Javier (1992). La eficiencia del sistema bancario español en la perspectiva europea. Moneda y crédito, No. 195, pp. 137-184.

Pérez, Francisco; Pastor, José Manuel (1994). La productividad del sistema bancario español (1986-1992). Papeles de Economía Española, No. 58, pp. 62-86.

Pérez, Francisco; Maudos, Joaquín; Pastor, José Manuel (1999). Sector bancario español (1985-1997): cambio estructural y competencia. Valencia, Instituto Valenciano de Investigaciones Económicas.

Pérez, Francisco; Maudos, Joaquín (2001), La eficiencia del sector bancario español en el contexto europeo. Economistas, No. 89, pp. 63-70.

Quesada, Javier (1994). Competitividad y eficiencia de las entidades financieras: análisis comparativo. Perspectivas del sistema financiero, No 48, pp. 111-122.

Revell, Jack (2000). Dimensión bancaria en la UEM. Papeles de economía española, No. 84-85, pp. 108-117.

Sanchis, Joan Ramon (2001), Dirección Estratégica de empresas cooperativas. Valencia, Edita Promolibro.

Sanchis, Joan Ramon; Palomo, Ricardo (1997). El Crédito Cooperativo en España. Valencia, Edita CIRIEC-España.

Sanchis, Joan Ramon; Campos, Vanessa (2007). Análisis estratégico de las secciones de crédito de las cooperativas agrarias en España. Evolución, situación actual y expectativas de futuro. Madrid, Confederación de Cooperativas Agrarias de España.

Santamaría, Rafael; Espitia, Manuel Antonio (1994). Estrategia competitiva y resultados de las cajas de ahorro en España. Ekonomiaz. Revista vasca de economía, No. 28, pp. 100-115.

Server, Ricardo; Melián, Amparo (1999). Estrategias competitivas y eficiencia de las secciones de crédito en la Comunidad Valenciana. Revista CIRIECEspaña No. 32, pp. 39-84. 
Server, Ricardo; Melián, Amparo (2001). Evaluación de la eficiencia de las entidades financieras en las secciones de crédito de las cooperativas. Revista de Investigación Agraria. Serie Producción y Protección Vegetales, No 16 (1), pp. 91-108.

Termes, Rafael (1994). La competitividad de la economía española y la eficacia del sis- tema financiero. Perspectivas del Sistema Financiero, № 48, pp. 31-43.

Tortosa, Emilio (2003). Bank cost efficiency as distribution dynamics: controlling for specialization is important. Investigaciones económicas, Vol. 27 (1), pp. 71-96.

Unión Nacional de Cooperativas de Crédito UNACC (2002-2007). Anuario de las cajas Rurales, Populares y Profesionales. Madrid. 